Monthly physical health reviews (including observations and weights), High Dose Antipsychotics Monitoring, Bloods and ECG records. After the initial baseline audit in Apr., 2019, some of the Quality Improvement (QI) approaches (4 PDSA cycles, driver diagrams, model for improvement) were used before conducting the re-audit in Oct., 2019.

Result. The baseline audit in Apr., 2019 showed 98\% compliance with physical assessment within 24 hours of admission, however, there was a significant gap in the monthly physical health reviews (62\%), Annual HIP (30\%), High-dose antipsychotic monitoring (10\%) and ECG/Bloods for antipsychotic monitoring (64\%) as per guidelines. 10 Female and 12 male patients had regularly refused obs, weight checks and physical health monitoring.

The re-audit showed an overall improvement of $92 \%$ in compliance, with increased High-dose antipsychotic monitoring (100\%), Monthly physical health clinics (88\%), Annual HIP (75\%), Annual antipsychotic monitoring/bloods/ECG(95\%).

Conclusion. Interventions, using QI approaches, between baseline and re-audit, included MDT discussion around strategies to improve patients' engagement with monthly physical health clinics with Specialty doctor, adding to care plan points, timescales and reminders in doctors' diaries for next bloods and ECGs due, MDT and patients' health education and a designated support staff for physical obs and maintaining physical health files. This helped in providing a framework to test recommended changes and evolve design based on repeated date collection between cycles.

The QI Interventions helped in implementation of a more holistic approach towards assessments due to which, the re-audit demonstrated a sustained improvement in compliance with all aspects of physical health monitoring.

\section{Re-audit of use of seclusion in a tier 4 adolescent psychiatric intensive care unit}

Yuki Takao*, Francesca Davis, Ivan Saeger, Sophia Ulhaq and Rafik Refaat

East London Foundation Trust

${ }^{*}$ Corresponding author.

doi: 10.1192/bjo.2021.319

Aims. To re-audit seclusion practices within a Tier 4 Adolescent PICU provision in London, originally audited in 2018. To ensure restrictive practices are only used in exceptional circumstances for short term risk management. To evaluate whether practice has improved following introduction of incidence reduction strategies and identify any further areas of development.

Background. This Tier 4 Adolescent PICU provides treatment of up to 16 high risk and unwell adolescents with severe and enduring mental health illnesses. Seclusion should be a short term risk management strategy with subsequent review of the care plan and treatment. It should be used for the shortest time possible. Following the audit in 2018, three strategies were implemented to reduce restrictive practice: (1) daily nursing safety huddles, (2) weekly Incidence Reduction meetings, and (3) ongoing QI project on restrictive practice.

Method. Data were collected from all patients requiring seclusion between January and December $2019(\mathrm{n}=18)$, which included 46 incidents. Data were collected from RiO computer records, extracting details of patient demographics, reasons and context of seclusion, risk reduction steps prior, length of seclusion, monitoring, and modifications to care plans.

Result. Average length of stay in seclusion was $20 \mathrm{~h}$, reduced from $30 \mathrm{~h}$ previously. Over half of patients requiring seclusion had symptoms of psychosis, consistent with the original audit. Majority of incidents involved assault to staff $(80.4 \%)$ as indication for seclusion, compared to $50 \%$ previously. In $58.7 \%$ of cases, verbal de-escalation was followed by further risk reduction with oral medication. Overall, rapid tranquillisation was required in $45.7 \%$ of incidents. Restraint was used in $84.8 \%$ of incidents, always in combination with at least one other management strategy.

Just under half of seclusions were monitored and documented in line with Trust guidelines, however, there was significant improvement in documentation of consultant reviews within $24 \mathrm{~h}$ from under $70 \%$ to over $90 \%$. Care plan modification rates improved from $63 \%$ to over $95 \%$.

Conclusion. Majority of seclusion incidents were due to violent acts by young people presenting with psychotic features/disorder. This reflects the complex nature of psychosis and the substantial need for research to reduce restrictive practice in such cases.

Ongoing review of data relating to seclusion will continue to inform and improve practice. This re-audit demonstrates improvement in various areas after implementation of strategies to reduce restrictive practice - importantly, average time in seclusion, documentation of 24 hour consultant reviews and focus on nonpharmacological risk reduction approaches in care plan modifications.

Audit of antipsychotic prescribing and monitoring for the management of behavioural and psychological symptoms of dementia

\section{Catrin Thomas ${ }^{1 *}$, Sharmi Bhattacharyya ${ }^{2}$ and Elizabeth Bond ${ }^{3}$}

${ }^{1}$ ST4 Old Age Psychiatry, Betsi Cadwaladr University Health Board; ${ }^{2}$ Consultant Old Age Psychiatrist and Clinical Lead, Betsi Cadwaladr University Health Board, Visiting Professor, University of Chester and ${ }^{3}$ Head of Pharmacy, Mental Health and Learning Disabilities Division, Betsi Cadwaladr University Health Board ${ }^{*}$ Corresponding author.

doi: 10.1192/bjo.2021.320

Aims. To assess the use of a piloted shorter version of the local Checklist for Antipsychotic Initiation and Review (CAIR) form by an Older Persons Community Mental Health Team (OPCMHT), and to assess whether the National Institute for Health and Care Excellence (NICE) guideline on use of antipsychotics for the management of behavioural and psychological symptoms of dementia (BPSD) is being adhered to.

Method. Retrospective audit analysing notes of all patients currently open to the OPCMHT that are prescribed an antipsychotic medication for the management of BPSD. Patients with a diagnosis of any subtype of dementia and prescribed any antipsychotic were included. Data collected from paper notes using an audit proforma.

Result. The total number of patients was 11 . The most common diagnosis was Alzheimer's disease (45\%), followed by mixed type dementia (36\%), vascular dementia (9\%) and Lewy Body dementia (9\%). The majority of the patients reside in their own home (64\%) whilst the remaining $36 \%$ reside in a residential home for the elderly and mentally infirm. The CAIR form was present in $73 \%$ of the patient's notes, however only $37 \%$ had the new, piloted, shorter version of the CAIR form. Of the CAIR forms present, only $63 \%$ were fully completed. There was documented evidence that $100 \%$ of patients had an assessment of underlying causes of their challenging behaviour; that non-pharmacological interventions were tried first; and that target symptoms were identified. There was evidence of a discussion with the patient or carer about the risks and benefits of antipsychotic use for all patients, however the details of the discussion was often vague. All patients had a review of the antipsychotic medication within the last three months.

Conclusion. There was evidence that pre-prescribing assessments are being undertaken for all patients. There needs to be clearer 
documentation of the discussions had with patients and carers about the risks and benefits of using antipsychotic medications for management of BPSD. A teaching session was held at the team meeting to highlight the risks and benefits. The team will ensure that they provide a health board approved leaflet to each patient and carer following their discussion. Only 73\% of the patients had a CAIR form in their notes and the team favour the original version. The team will revert back to using the original version of the CAIR form as it has more space allocated to document ongoing reviews. We will re-audit in 6 months time.

\section{On-call medical seclusion reviews: are we meeting MHA code of practice (COP) requirements? \\ Oliver Turner ${ }^{1 \star}$ and David Leung ${ }^{2}$ \\ ${ }^{1}$ Leeds and York Partnership NHS Foundation Trust and ${ }^{2}$ PICU, Newsam Ward 1, Leeds and York Partnership NHS Foundation Trust ${ }^{*}$ Corresponding author.}

doi: 10.1192/bjo.2021.321

Aims. Are Junior Trainee, Medical Seclusion Reviews complaint with MHA COP Criteria?

Objectives. Are we seeing newly secluded patients on time?

Are we documenting these reviews in clinical notes?

Do documented reviews meet criteria stated by the MHA COP 26.133 ?

Are we informing Higher Trainees of the need for MDT reviews? Background. Seclusion is an important aspect of inpatient care. MHA COP Chapter 26 provides guidance for documenting seclusion reviews, ensuring safeguards are in place to protect patient's safety and human rights. Secluded patients require a medical review within 1 hour, and four hourly thereafter, until a higher trainee or Consultant undertake an MDT Review. In our Trust, LYPFT, trainees undertake these reviews. There is noted discrepancy in seclusion review documentation. This audit identifies our compliance with time limits, and whether documentation meets the required criteria in the MHA Code of Practice

Method. Our Sample includes all Out-of-Hour Junior Trainee Medical Seclusion Reviews between 01/01/20 and 01/04/20 at LYPFT. Seclusions were identified from on call logs, and clinical notes were reviewed for a documented seclusion review. The date and time of seclusion are recorded, whether a 1 or 4 hourly review, and the time of review. We recorded any mention of: physical health; mental state; observation levels; recent medication; medication side effects; risk to others; risk to self and the need for ongoing seclusion. Result. 56 episodes of seclusion were identified; all 56 had a documented medical seclusion review. 49 reviews were on time, 4 were late with a documented reason, and 3 were late without. There was documentation of the Higher Trainee being informed in 53 reviews.

No seclusion reviews mentioned all MHA COP criteria. We more frequently mentioned patients' physical health (51), psychiatric health (52) and need for seclusion (54). 46 seclusion reviews mentioned risk of harm to others; only 3 mentioned risk of self-harm. 25 seclusion reviews mentioned medication, and 5 mentioned review for side effects. 5 seclusion reviews mentioned observation levels.

Conclusion. Our Junior Doctor Seclusion Reviews were not meeting the MHA Code of Practice Criteria, and we believe this to largely be due to lack of awareness of the standards. As such, results have been disseminated to Junior trainees in weekly teaching. We created a medical seclusion review template, adopted by the Trust, to ensure documentation compliance with the MHA COP. Junior doctor inductions now include a presentation regarding Seclusion, the reviews and documentation. We will re-audit in 12 months.
Early intervention in psychosis team (EIT): pathways to care

Chloe Uffendell ${ }^{\star}$ and John Stevens

MerseyCare NHS Trust

${ }^{\star}$ Corresponding author.

doi: 10.1192/bjo.2021.322

Aims. The main aim of this study was to investigate whether the EIT access and waiting time standard ( $>60 \%$ of people experiencing first episode psychosis (FEP) are treated with a NICE-approved care package within two weeks of referral) was being met within Liverpool EIT.

We also wanted to understand the pathway to treatment within EIT services, identify delays in the process of triage/assessment/ $\mathrm{MDT} / \mathrm{medical}$ review and implement changes to reduce delays.

Method. This study was a retrospective cross-sectional audit of all patients accepted on to the FEP pathway following MDT discussion in the Liverpool EIT Teams across May and June 2020.

Case notes were analysed for delays in referral, engagement with assessment and care-coordinators, as well as prescriber review offering medication. The data were collated and analysed before implementing changes.

Result. 40 patients presented as FEP in May and June 2020, 6 were excluded due to an extended inpatient stay.

Within the remaining patient cohort $(n=34), 64.7 \%$ of patients were engaged with a care package within 14 days. Only $14.7 \%$ of patients received an offer of medication within 14 days, the mean time to be offered medication was 39 days.

$26 \%$ of patients first contact within MerseyCare Trust was with EIT, $74 \%$ presented elsewhere. $24 \%$ instead presented to liaison psychiatry from A\&E departments, $18 \%$ to the single point of access team, 9\% to criminal justice liaison team (CJLT) and 9\% to North West Ambulance Service triage car.

$29 \%$ of referrals came from the community (GP and counselling services), $15 \%$ from CRHT (crisis resolution and home treatment team), $14 \%$ from CJLT, $12 \%$ from urgent care team, $9 \%$ from liaison psychiatry.

Conclusion. The Access and Waiting time standard was met. However, this study showed that patients were not being referred to EIT at first point of contact. This study shows $26 \%$ of service users first presented to liaison psychiatry, yet only $1 / 3$ of those were immediately referred to EIT, the remainder being later referred by other services e.g. CRHT.

In addition to referral delays, lack of medical practitioner availability caused significant delays in arranging medical reviews, delaying patients access to medication.

The changes implemented to address these issues included educating MerseyCare services in the early recognition of psychosis to increase early referral. Non-medical prescribers' roles were developed to perform initial medical reviews in addition to doctors, allowing patients earlier medication access. This allowed 'urgent slots' to be developed, time set aside for emergencies enabling prompt review of urgent cases.

An audit of lithium prescribing practices in an old age psychiatry service highlighting renal impairment in this cohort

Leia Valentine*, John Cannon, Siobhan Marmion, Michelle Corcoran, Marguerite Cryan, Geraldine McCarthy and Catherine Dolan

Liscarney House, Psychiatry of Old Age Service

${ }^{\star}$ Corresponding author.

doi: 10.1192/bjo.2021.323 